\title{
(1)(@) Gestión de la calidad en el sector de telecomunicaciones. Orientaciones hacia la mejora continua en la Corporación Nacional de Telecomunicaciones, Azogues - Ecuador.
}

Quality Management in the telecommunications sector. Orientations towards continuous improvement in the National Telecommunications Corporation, Azogues - Ecuador.

Alicia Mariela Torres Beltrán ${ }^{1}$, Kléber Antonio Luna Altamirano. ${ }^{2}$, Jorge Edwin Ormaza Andrade $^{3}$ \& Marcos Eduardo Cantos Ochoa. ${ }^{4}$

\section{DOI: https://doi.org/10.33262/visionariodigital.v3i2.407}

\section{Resumen.}

La investigación se plantea como objetivo analizar la gestión de la calidad del servicio en el sector de comunicaciones, estudiando específicamente el caso de la Unidad Comercial de la Corporación Nacional de Telecomunicaciones (CNT EP), ubicada en la Ciudad de Azogues, Ecuador. La idea es a partir de los resultados obtenidos direccionar acciones para la mejora continua en la unidad estudiada. La investigación es de tipo descriptiva con un enfoque netamente cualitativo; es no experimental y de campo, se utiliza como técnica de recolección de información empírica la encuesta y entrevista a clientes y funcionarios de la corporación. Los resultados indican fallas por la obsolescencia y saturación de la red, ocasionando baja velocidad en el servicio y obstáculos para la empresa; incidentes que no fueron atendidos de forma apropiada y eficiente por Unidad Técnica, encargada de la atención de daños, esto reduce la calidad del servicio prestado y conlleva a proponer un modelo de mejora continua para el servicio de internet de la Corporación Nacional de Telecomunicaciones CNT EP; esto a partir de la migración del servicio de internet fijo por cobre a la red de fibra óptica. Con esto se presume se reduzca el número de reclamos, y se incremente el nivel de satisfacción de los usuarios. Lo

1 Universidad Católica de Cuenca, Maestrante en Administración de Empresas, Cuenca, Ecuador. amtorresb420@psg.ucacue.edu.ec

2 Universidad Católica de Cuenca, Unidad Académica de Administración, Cuenca, Ecuador. klunaa@ucacue.edu.ec

3 Universidad Católica de Cuenca Sede Azogues, Unidad Académica de Administración, Azogues, Ecuador.jormaza@ucacue.edu.ec

4 Universidad Católica de Cuenca, Unidad Académica de Administración, Cuenca, Ecuador. mecantoso@ucacue.edu.ec 
anterior de la mano de un proceso de contratación de personal eficiente y un programa de capacitación que permita brindar soporte técnico a los usuarios.

Palabras clave: Gestión de calidad, calidad de servicio, Corporación Nacional de Telecomunicaciones.

\begin{abstract}
.
The research aims to analyze the management of service quality in the communications sector, specifically studying the case of the Commercial Unit of the National Telecommunications Corporation (CNT EP), located in the city of Azogues, Ecuador. The idea is from the results obtained to direct actions for continuous improvement in the unit studied. The research is of a descriptive type with a clearly qualitative focus; it is non-experimental and field-based; the survey and interview of clients and corporate officials is used as a technique for collecting empirical information. The results indicate failures due to the obsolescence and saturation of the network, causing low speed in the service and obstacles for the company; incidents that were not attended in an appropriate and efficient way by the Technical Unit, in charge of the attention of damages, this reduces the quality of the service provided and leads to propose a model of continuous improvement for the internet service of the National Telecommunications Corporation CNT EP; this from the migration of the fixed internet service by copper to the fiber optic network. This is presumed to reduce the number of complaints, and increase the level of user satisfaction. This is accompanied by an efficient recruitment process and a training program to provide technical support to users.
\end{abstract}

Keywords: Quality management, quality of service, National Telecommunications Corporation.

\title{
Introducción.
}

A nivel mundial, el sector de las telecomunicaciones ha avanzado de forma vertiginosa y sorprendente, como consecuencia de la diversificación de productos y servicios que se ofrecen y se orientan a satisfacer las necesidades de comunicación de la población. Los constantes cambios a los que ha sido expuesto han exigido respuestas ágiles para hacer frente a circunstancias que se han presentado a lo largo del tiempo; por ello, resulta de gran importancia, disponer de herramientas útiles, de fácil aplicación, que permitan estar a la vanguardia de la tecnología y la innovación.

En el entorno latinoamericano, el sector de telecomunicaciones ha sufrido grandes transformaciones durante las últimas décadas, cambios tecnológicos y requerimientos por parte de clientes han sido cada vez más exigentes, requiriendo contar con servicios y productos de calidad que permitan alcanzar un posicionamiento en el mercado, y por ende poder lograr un desenvolvimiento dentro de la industria, que apuesta a la concreción de los mejores resultados posibles. 
La aparición de nuevas tecnologías ha provocado que se incremente la competencia entre oferentes de los servicios de telecomunicaciones, surgiendo nuevas formas de comunicación entre personas, organizaciones, y organismos y sistemas diversos, convirtiéndose la comunicación en eje fundamental para la correcta marcha y funcionamiento de las organizaciones, sean estas públicas, privadas o cuya naturaleza represente la combinación de otras modalidades.

En todo caso, la comunicación en tiempo real, se constituye en elemento esencial para permanecer en mercados altamente globalizados y con grandes exigencias; ejemplo de ello, lo representa la empresa Mexicana Telmex quien producto de sus avances ha logrado sacar del mercado a sus competidores principales. Según plantea Huerta y Gómez (2013): "la ley de la oferta y la demanda, así como la experiencia de los dueños deben determinar el rumbo de las industrias de los medios de comunicación y las telecomunicaciones, así como sus mercados" (p.114).

Hoy en día las comunicaciones se encuentran dentro de un mercado globalizado, y al liberalizar la prestación que ofrece este mercado, no es solo prioridad del Estado “(...) han pasado de ser un deber de suministro estatal, a convertirse en una mercancía, sin embargo, no se trata de una mercancía cualquiera, debido a que los servicios de telecomunicación se encuentran en la base de nuestra moderna sociedad de la información" (Schneider, 2016, p.215).

Por lo expuesto, la creación de empresas requeridas para la prestación de estos servicios, sean de orden público o privado, deben garantizar el acceso a estos servicios incluyendo las localidades donde no se obtiene una rentabilidad económica sino más bien una rentabilidad social.

En lo que respecta a esta temática en la sociedad ecuatoriana, en los últimos años, se han suscitado cambios profundos, pasando de tener una tecnología obsoleta, a contar con tecnología de punta, que permite competir no solo a nivel nacional, sino internacional con cualquier compañía que oferte servicios de telecomunicaciones. Se ha adaptado infraestructura tecnológica para cubrir requerimientos por distancias y capacidad, que hasta hace poco tiempo, hubiera resultado imposible otorgar.

En este caso, el Estado ecuatoriano, encargado de prestar este tipo de servicios de telecomunicaciones a los habitantes del país de manera directa, ha trabajado en aras de ofrecer calidad, mediante el uso de tecnologías modernas que permitan alcanzar la satisfacción de los usuarios con tarifas accesibles. Sin embargo, en la sociedad ecuatoriana, la gestión pública carece de una administración eficiente y eficaz que conlleve a complacer las necesidades reales de los ciudadanos, a la optimización de recursos, y hacia la obtención de niveles adecuados de productividad, restando con ello eficiencia y eficacia en la creación de servicios de mayor calidad y con el menor costo posible. 
Este hecho, impone a la institución pública que actualmente ofrece estos servicios, a contar con tecnologías de punta, innovación constante en los procesos, brindar el servicio con calidad, siendo mejor que el otro, ser competitivos, ser reconocido, y permanecer posicionado en la mente del consumidor, a fin de incrementar el número de usuarios dentro de esta economía.

El acceso a las telecomunicaciones e información, deben ser igual en calidad y precio para los consumidores de zonas urbanas y rurales; es decir, sin discriminación a la conservación y desarrollo del servicio. Se busca entonces una finalidad social, que evite las clases sociales: ricos y pobres de acuerdo a su poder comunicativo. En cuanto a la noción del servicio universal.

Ante estos planteamientos, la Corporación Nacional de Telecomunicaciones (CNT EP), debe enfocarse en otorgar un servicio competitivo mediante la aplicación de estrategias comerciales y estrategias de mejora continua que potencien su calidad constantemente, más aún cuando estos servicios móviles están dominados por la empresa privada. La importancia radica en la definición de los servicios de telecomunicaciones a nivel público de manera eficiente y con ventajas competitivas.

Ahora bien, la búsqueda del mejoramiento de la calidad en los servicios públicos que se ofrece a nivel regional, no es reciente, Torres, citado por Bernal, Pedraza, y Sánchez (2015) aseveran: “A partir de la década de los noventa, la calidad de los servicios se ha convertido en un principal elemento para cumplir con los objetivos y regulaciones gubernamentales para satisfacer las expectativas de una ciudadanía cada vez más informada y exigente" (p.12). Por lo que, una correcta gestión de calidad de los productos y de los servicios que ofrezca la Unidad Comercial de la Corporación Nacional de Telecomunicaciones (CNT EP) en la ciudad de Azogues, generará ventajas competitivas sobre la misma en doble sentido; por un lado, con un efecto positivo sobre las ventas, y por otro, la posible reducción de los costos a largo plazo.

Un servicio será de calidad, no sólo si se ofrece un buen servicio, sino al satisfacer o exceder las expectativas del cliente; esto incluye buena atención y la buena imagen que se proyecta hacia el consumidor final. Los objetivos de la institución deben estar encaminados la calidad plena y en consecuencia a la satisfacción del cliente a través de la aplicación correcta de los procesos y de su sistema de gestión de calidad. Así se hace necesario la participación, responsabilidad, perfección y el espíritu del servicio como existe diferencias en los gustos y preferencias, será necesario trabajar para obtener un nivel esperado de los bienes que consume el cliente o el consumidor final, a un precio accesible.

Para el caso ecuatoriano, los sectores económicos no se excluyen de la aplicación de modelos de gestión de la calidad recomendados a nivel mundial y de Latinoamérica, es 
necesario alinearse a las tendencias que se presentan, y a su vez viabilizar el cumplimiento de objetivos empresariales orientados a la calidad, y por ende a la satisfacción del cliente.

Ahora bien, la necesidad de mantener y actualizar el sistema de gestión de calidad y operar bajo un esquema de mejora continua, para satisfacer las exigencias de los clientes, asegurando un crecimiento y rentabilidad permanente del negocio, así como el desarrollo profesional de los empleados debe ser una exigencia diaria. En este sentido, esta investigación, se plantea como objetivo central analizar la gestión de la calidad del servicio en el sector de comunicaciones, estudiando específicamente el caso de la Unidad Comercial de la Corporación Nacional de Telecomunicaciones (CNT EP), ubicada en la Ciudad de Azogues, Ecuador.

Desde la perspectiva metodológica, la investigación es de orden cualitativa, de tipo descriptiva, no experimental y de campo. Se apoya en fuentes primarias, representadas por informantes clave de la empresa en estudio, así como en fuentes secundarias. Para la elaboración del marco teórico se empleó el criterio de varios autores y se efectúa una síntesis de estos criterios, tomando en cuenta los aspectos más relevantes para realizar aportaciones al conocimiento científico derivado, complementando con otras fuentes de carácter secundaria, como lo es la información contenida en reportes e informes de la CNT EP, Constitución del Ecuador, Plan Nacional de Desarrollo para el período 20172021, Ley de Comunicación, entre otros documentos.

Se diseñaron cuestionarios y guion de entrevista, aplicándose mediante técnicas como la encuesta y la entrevista estructurada. Esto permitió ahondar el análisis de las variables y agregar validez y fiabilidad a la investigación. La población estuvo conformada la Unidad Comercial de la Corporación Nacional de Telecomunicaciones de la Corporación Nacional de Telecomunicaciones (CNT EP), siendo sus informantes claves directivos, trabajadores y clientes que reciben el servicio de internet fijo residencial por cobre en la parroquia Javier Loyola perteneciente a la Ciudad de Azogues, de la empresa. La Corporación Nacional de Telecomunicaciones (CNT EP) cuenta con 512 usuarios del servicio de internet fijo residencial por cobre, quienes constituyen la población de la presente investigación, a partir de esta información obtenida en la empresa, se halla la muestra estadística para la aplicación de la encuesta a los clientes.

La información recolectada de fuentes primarias, es decir, del lugar objeto de investigación en la Parroquia Javier Loyola de la ciudad de Azogues; por un lado, personal de los departamentos de la CNT EP, de manera especial y puntual a la Unidad Comercial; y por el otro clientes y usuarios del servicio de internet de la Parroquia Javier Loyola.

Con respecto a la técnica de la entrevista, la misma que para Taylor y Bogdan (como se citó en Quecedo y Castaño, 2002) se realizaron reiterados encuentros cara a cara entre el investigador y los informantes, encuentros dirigidos a la comprensión de las perspectivas 
que tienen los informantes de sus experiencias o situaciones, tal como las expresan con sus propias palabras". El beneficio de esta técnica para la formulación de la propuesta radica por la forma espontánea y flexible para obtener información. Las entrevistas estuvieron dirigidas hacia los directivos de la Corporación Nacional de Telecomunicaciones CNT EP en la Ciudad de Azogues, así como a los funcionarios que pertenecen a la Unidad Comercial, asimismo, se realizaron entrevistas clínicas a varios colaboradores de diferentes departamentos de la empresa.

\section{Gestión de la calidad: Reflexiones teóricas}

Desde la antigüedad, la calidad ha sido considerada muy importante en todos los aspectos del diario vivir; es así como, constantemente se pretendía que los objetos utilizados para la caza, la pesca, la agricultura, entre otros, ofrezcan mayor durabilidad y satisfagan de mejor manera las necesidades de quien los utilizaba. En el mismo sentido, en el sector de la construcción buscaban que las edificaciones proporcionen seguridad a quienes habitaban en la misma, que su estructura fuera de calidad, es decir, resista las inclemencias del tiempo. Por otro lado, la pretensión de producir u obtener bienes o servicios de calidad no es reciente, ha sido un elemento consustancial con el hombre e inherente a todas las actividades que éste ha realizado desde el momento que ha llegado a la faz de la tierra.

En el ámbito gerencial, la evolución cronológica de la calidad se origina a inicios de administración científica, concretamente con el control de la calidad por inspección de la producción, que da comienzo formal al concepto de calidad. En un segundo momento se tiene el aseguramiento de la calidad a través de la gestión en los procesos, más no a las funciones, desde este momento, cobra importancia la organización horizontal, desechando las jerarquías verticales.

En la lectura revisada, se confirma que autores de diversas regiones del mundo han generado conocimiento y han socializado los resultados alcanzados por la aplicación de modelos, estrategias, metodologías y herramientas sobre la calidad y su gestión dentro de las empresas. Así mismo, los servicios, productos, e iniciativas que han sido propagados tanto al interior de la academia, como en las organizaciones, han servido para profundizar o iniciar estudios, y poner en práctica y propender la calidad como principios rectores de las operaciones de una empresa.

En una inquietante sociedad actual, inminentemente dominada por la presencia vertiginosa de cambios en todos los ámbitos del convivir, nuevos enfoques empresariales son requeridos para enfrentar nuevas realidades y exigencias a nivel de productos y servicios. En el mundo de los servicios, las ideas de la calidad no deben vincularse sólo con la tenencia de recursos tecnológicos, el simple hecho de no recibir servicios ajustados a las necesidades de los clientes conduce a las organizaciones fracaso es seguro (Ajá, 2002). 
En este orden de ideas, se resalta que, durante las últimas décadas del siglo $\mathrm{XX}$, surge con fuerza en el mundo empresarial un paradigma que ha tenido como centro el concepto de calidad, cuyo impacto se dio inicialmente dentro de la industria, pero en lo posterior se difundieron y popularizaron en otros sectores como el financiero, salud, social, por nombrar algunos.

La difusión de este paradigma de la calidad, trajo consigo la difusión de una variedad de conceptos, métodos y modelos de gestión de diversa índole entre los que destacan: circulo de calidad, sistemas de aseguramiento de la calidad, gestión de la calidad total, normas ISO 9000, entre otros modelos y metodologías como el six sigma.

La implementación de la calidad en las organizaciones, busca garantizar el producto o servicio a la población a fin que estas satisfagan las necesidades a todo nivel, con un grado predecible de uniformidad, fiabilidad, a un bajo costo que se adecue a las necesidades del mercado. La satisfacción del cliente a través de productos y servicios que cumplan con estándares de calidad aceptados, permitirá a la empresa competir en mercados exigentes, buscando un mejoramiento continuo, de agrado a los clientes, estandarización y control de procesos, con lo que se obtiene herramientas prácticas para una gestión integral, que permitan obtener un mayor rendimiento en cuanto a funcionamiento y durabilidad.

Sin duda, la calidad es una herramienta básica e indispensable que debe estar presente en todos los procesos, productos y servicios de una organización o empresa, que se podrá lograr con la participación de todos quienes conforman la institución o unidad empresarial. Sus resultados repercutirán positivamente tanto a clientes como a la misma empresa o ente institucional, más aún, que, si las motivaciones de las organizaciones por implementar la calidad es mejorar su imagen dentro del mercado y, mejorar la organización y gestión de la empresa que provoque una mayor satisfacción al cliente, demuestra el interés de las empresas por lograr un reconocimiento sobre todo externo.

En este sentido, al conceptualizar la calidad, vienen implícitas características a nivel de los producto o servicio, que deben cumplir con una necesidad o expectativa establecida, que por lo general son implícitas u obligatorias. La calidad se valora como el hecho de satisfacer las expectativas, con sentido de excelencia simultáneos (Parasuraman, Zeithaml, y Berry, 1985). La calidad no es una ventaja competitiva en sí misma, es simplemente un requisito.

Entre tanto, los atributos que se asocian a la calidad como: el precio y plazo de entrega, confiabilidad, durabilidad, mantenimiento, aspectos físicos, etc., son requisitos para sobrevivir y determinar ventajas competitivas. No obstante, la calidad constituye una ventaja significativa, que asegura posicionamiento y permanencia en el mercado, mejora las utilidades y por consiguiente los resultados de la empresa en el mediano y largo plazo, pudiendo convertirse en un estilo de vida. Para Gutiérrez (2004), la calidad existe cuando los miembros de la institución poseen, comparten y ejercen una serie de valores cuyo fin 
último es la satisfacción de las necesidades del cliente. El autor destaca el compromiso y responsabilidad de los miembros que conforman una institución, a fin que, con su empeño en el trabajo, se propugne la perfección y se pueda satisfacer las necesidades de todos los clientes.

Así la calidad, puede responder a una dimensión subjetiva y ambigua, que no puede ser fácilmente medible, ni demostrable, más aún a nivel de los servicios, cuyas características denotan intangibilidad, heterogeneidad, carácter perecedero y la simultaneidad entre la producción y consumo simultáneos (Parasuraman, Zeithaml, y Berry, 1985).

En lo que respecta a la calidad en los servicios, los esfuerzos referidos a su consolidación, hacen alusión, por un lado, al talento o recurso humano, enfocando el esfuerzo de las personas que conforman la organización. El personal asume responsabilidades de: ejecutar, monitorear y evaluar el sistema de gestión, convirtiéndose en obligación de los entes empresariales invertir en formación, capacitación y actualización de su personal; y por el otro, a recursos técnicos, herramientas, tecnologías, máquinas, que apoyan al primer esfuerzo (recurso humano) para que de forma eficiente y eficaz se pueda cumplir con las obligaciones encomendadas.

En el caso de los servicios, estos pueden ser manejados desde diferentes puntos de vista; pudiendo afirmar que cada persona tiene su propio concepto, diferentes entre sí, pero seguramente válido. Peña (1992) sostiene: "la gerencia de servicio como (...) una economía donde las relaciones son más importantes que los productos y, para competir a nivel de la calidad, se debe hacer una adecuada fijación de estándares ya que la empresa es un equipo que brinda una garantía de calidad al cliente" (p.65).

Sin duda, en la actualidad la pirámide está invertida, y el cliente es lo primero, por lo tanto, es imprescindible hacer que, desde el último de los trabajadores, sienta que es parte de un compromiso de la institución, por lo tanto, su obligación con el rol que desempeña está dirigida a satisfacer al cliente y por ende a la empresa o ente donde labora.

Por lo tanto, el servicio al cliente constituye el servicio o atención que el personal de la empresa brinda a sus clientes y usuarios, durante las actividades de consultas, reclamos o ventas presentados por los diversos medios destinados para el efecto. El servicio recibido por el cliente, será evaluado de acuerdo a su percepción, ya sea de forma positiva o negativa; y, del resultado de esta apreciación recomendará o no la utilización de productos y servicios que brinda la empresa.

En este orden de ideas, precisando lo que implica la gestión de la calidad en una organización, se alude al desarrollo de una serie de procesos y estrategias que direccionan las organizaciones, sean de servicios públicos o privados para cumplir las expectativas de valor exigidas por los clientes (Parasuraman, Zeithaml, y Berry, 1985). Ajá (2002) afirma: "la calidad es un conjunto de actividades coordinadas para dirigir y controlar una 
organización en materia de calidad. Para el autor, la gestión de la calidad se orienta a coordinar tanto actividades de las personas como de los recursos para mejorar lo que oferta a sus clientes o usuarios" (p.3).

La administración de la calidad, aceptada también como gestión de la calidad está orientada a crear conciencia de la calidad en todos los procesos de una organización y es utilizada por diversos sectores del área pública como privada. Ahora bien, la implementación de un sistema de gestión de calidad, conlleva a la organización a desarrollar procesos conducentes, a identificar las características relevantes del producto o servicio que demandan y exigen los clientes (calidad, el precio, y otras propias del producto o del servicio), el cumplimiento de estas propiedades logrará sin duda, aumentar el porcentaje de ventas estimadas por las empresas o las organizaciones.

Un sistema de Gestión de la Calidad es una directriz, que orienta el cumplimiento de los objetivos y prioriza la mejora continua en el desempeño y la eficacia de los procesos que desarrollan todos los miembros de la organización para ser más competitivos en el mercado, obtener rentabilidad y satisfacción de las necesidades de los clientes. Para un buen desarrollo de gestión de la calidad las empresas deben contar con los siguientes elementos: Una estructura organizacional, una planificación estratégica, recursos tanto materiales como humanos, desarrollo de procesos, y procedimientos.

No obstante, entre los beneficios que la empresa percibirá al contar con un sistema de gestión están la identificación de acciones preventivas y correctivas que permitan desarrollar una administración eficiente, mejorando la motivación y comunicación con sus empleados, mejores resultados, posicionamiento en el mercado, fidelización de clientes satisfechos y ventaja competitiva ya que se logra un posicionamiento en el mercado. Por otra parte, toda ventaja competitiva constituye una estrategia que rivaliza en el mercado internacional; donde, los factores de producción: tierra, trabajo y capital, determinaban el precio de un producto, así como la oferta y la demanda. Actualmente el capital intelectual, las tecnologías de la información y comunicación (TIC) son los factores competitivos que determinan la ventaja competitiva.

James (1997) explica ciertas funciones dentro de la gestión de calidad, entre ellas: planificación, organización, dirección, personal y control. La planificación siempre camina hacia el futuro llegando a cumplir con las metas planteadas por la entidad, al mismo tiempo brinda la capacidad de ser dinámico anticipándose a eventos futuros, determinando las acciones esenciales para desafiar de manera positiva a estos acontecimientos, siendo primordial la eficiencia dentro de un proceso de mejora de la calidad. Los elementos clave que incluye son: análisis del entorno, misión de la calidad, establecimiento de la política de calidad, objetivos estratégicos de calidad, y planes de acción de la calidad, James (1997). 
Ante estos planteamientos existen modelos de gestión de calidad que asumen como elementos centrales: el direccionamiento estratégico que implica concebir la calidad desde el nivel estratégico de la organización, es decir definir elementos estratégicos como la misión, visión, valores, políticas, objetivos y elementos de gestión que lleven implícito la calidad como principio rector y conducente de las operaciones desarrolladas en las organizaciones. Todo ello, apostando a la mejora continua de las operaciones de servicios, desde las responsabilidades de la dirección y la gestionan que estos realicen de los recursos disponibles para ofrecer servicios que cubran las necesidades y expectativas de los clientes. Los planteamientos anteriores, establecen orientaciones para el diseño de un modelo de gestión de la calidad en cualquier organización, sea del sector privado o público.

\section{Corporación Nacional de Telecomunicaciones CNT EP: Un análisis desde la gestión de la calidad del servicio}

En el año 1884, el Ecuador inicia sus comunicaciones mediante la transmisión telegráfica alámbrica de mensajes, es así como, luego de varios años se crean empresas que brindan servicios de telefonía para las principales ciudades como lo son Quito y Guayaquil, extendiéndose posteriormente a otras provincias. En el año de 1968, se crea la empresa de Telecomunicaciones, Agua Potable, Alcantarillado y Saneamiento de Cuenca, quien inicia con sus servicios de telefonía, agua potable y alcantarillado; en la actualidad, además de los servicios referidos la empresa municipal ha extendido los servicios de internet y televisión.

En 1972 aparece el Instituto Ecuatoriano de Telecomunicaciones (IETEL), que hasta 1992 regulaba las redes telefónicas en el Ecuador, el Instituto brindaba los servicios de telefonía fija, y estuvo integrado por las empresas de teléfonos tanto del norte como del sur del país. Para 1992 transformarse en EMETEL, luego en EMETEL S.A. Empresa Autónoma de Telecomunicaciones para en el año 1997, dividirse en dos nuevas empresas estatales: Andinatel S. A. y Pacifictel S. A., estas últimas encargadas de brindar servicios de telefonía e internet.

Cronológicamente, el 30 de octubre de 2008, se crea la Corporación Nacional de Telecomunicaciones CNT S. A., producto de la fusión entre las empresas Andinatel S. A. y Pacifictel S. A. la cual para el año 2010 se convierte en empresa pública y en el principal proveedor de servicios de telecomunicaciones de la sociedad ecuatoriana. La CNT EP. La empresa tuvo que afrontar demandas por parte de los empleados de Pacifictel, viéndose la necesidad de crear un departamento jurídico para afrontar esta situación.

Su presencia es nacional, y lo hace a través de oficinas ubicadas de manera estratégica en cada una de las provincias. Su objetivo, satisfacer la demanda nacional de servicios de telecomunicaciones. En la Provincia del Cañar, la Corporación Nacional de Telecomunicaciones CNT EP cuenta con un centro integrado de servicios CIS en la 
capital provincial Azogues donde se encuentra la administración provincial; y, cuatro puntos de atención en Biblián, Cañar, El Tambo y La Troncal.

Según información de la empresa, ésta cuenta con ejes estratégicos sobre los cuales fundamenta su dirección, integrados por misión, visión, valores estratégicos. La empresa se preocupa por alcanzar sus objetivos en el corto, mediano y largo plazo, y servicio de responsabilidad social. La Corporación Nacional de Telecomunicaciones se preocupa de la calidad en los procesos y la prestación del servicio, desde hace varios años cuenta con un modelo de gestión de la calidad, mediante la certificación de la Norma ISO, mantiene implementada la versión 9001:2015.

La Unidad Comercial de la Corporación Nacional de Telecomunicaciones -CNT EP- de la ciudad de Azogues, provincia del Cañar, creada en el año 2008, se dedica a prestar servicios de telecomunicaciones en Ecuador. Su portafolio de servicios se compone de: telefonía fija, telefonía móvil, internet fijo y móvil; y, televisión satelital.

Al analizar la corporación desde la perspectiva interna, se realizaron una serie de entrevistas a personal clave de la misma, pudiendo detectar debilidades asociadas a la gestión que actualmente desarrolla. En este sentido, se escuchó de los clientes y usuarios que la calidad de servicio ofrecida es deficiente, los empleados o colaboradores no satisfacen necesidades y expectativas, presentando los mismos falta de compromiso y de interés, aunado a ello, los usuarios manifestaban que el precio de los servicios es elevado (Entrevistas realizadas, 2018).

Con la información obtenida, conllevó a analizar elementos internos, con el propósito de verificar a nivel interno elementos estratégicos de la empresa, entre los que destacan: misión, visión, valores, políticas, objetivos, así como otros elementos de gestión entre los que recalcan las responsabilidades de la dirección y la gestión que estos realizan de los recursos utilizables.

Estos aspectos, se analizan en la corporación con la finalidad de precisar la calidad como parte esencial de los elementos estratégicos de la empresa estudiada, buscando estructurar un modelo de gestión de calidad orientado a la mejora continua y la satisfacción de las expectativas y requerimientos de clientes y usuarios.

En este sentido, entre los elementos de gestión a precisar en la CNT EP, la cual declara siete objetivos de calidad que forman parte del proceso de Planificación Estratégica 2014 - 2018 y que corresponden a sus objetivos empresariales y son: (CNT EP, 2017)

- Incrementar ingresos.

- Incrementar cobertura banda ancha en instituciones sociales.

- Incrementar los niveles de fidelización, retención y satisfacción de los clientes.

- Incrementar base de clientes en las líneas de negocio.

- Incrementar la efectividad gestión de reclamos. 
- Incrementar la disponibilidad de las redes de telecomunicaciones y plataformas TI.

- Incrementar la efectividad de los procesos de venta, instalación.

Estos objetivos se consideran estratégicos para la Corporación, sin embargo, es evidente que se debe fortalecer lo concerniente a la calidad total en las operaciones emprendidas.

Con respecto a la misión, expresa: "Brindamos a los ecuatorianos la mejor experiencia de servicios convergentes de telecomunicaciones y TICs, para su desarrollo e integración al mundo, impulsando el crecimiento de nuestra gente y creando valor para la sociedad" (CNT EP, 2017). Es evidente la no inclusión de la calidad como parte central de la razón de ser de la corporación, aspecto esencial, que sebe declararse desde este direccionamiento central de la empresa.

A pesar de ello, en algunas áreas estratégicas de la corporación, como son el área de post venta, servicio al cliente y el área de bodegas, se incluye como parte de la misión, la ejecución de procesos de manera eficiente y apegado a las normativas existentes; se resalta la comunicación, el enfoque en los resultados, el trabajo en equipo, la integridad, la orientación al servicio, el pensamiento analítico, disposición a aprender, negociación y manejo de conflictos, reconocimiento de problemas, diligencia y la calidad de trabajo. En estas áreas, se resalta la calidad como parte de las operaciones desarrolladas y como competencia básica que orienta el trabajo desarrollado.

Con respecto a la visión, la corporación declara: "Ser la empresa líder de servicios convergentes de telecomunicaciones y TICs del Ecuador, a través de la excelencia en su gestión, el valor agregado y la experiencia que ofrece a sus clientes; que sea orgullo de los ecuatorianos" (CNT EP, 2017). Es evidente como la calidad no resalta en las afirmaciones establecidas como parte de la visión organizacional.

Asimismo, la corporación declara como valores: compromiso con el cliente, trabajo en equipo, eficiencia, innovación, integridad y la responsabilidad social (CNT EP, 2017), sin precisión de valores asociados con la calidad total de las operaciones, en todo caso, podría asociarse la calidad con el compromiso con el cliente, en cuanto a darle al cliente lo que requiere, satisfaciendo sus expectativas, necesidades y deseos.

La organización declara de igual forma su política, precisando lo importante de la política de la calidad definida, su comunicación, comprensión y aplicación de la misma, orientados a su aplicación a fin de obtener la satisfacción de los clientes; ante esto, en función de la investigación realizada, es evidente que la política de la calidad no es aplicada por todos los colaboradores de la empresa. Ante esta situación, se deben realizar talleres donde se socialice la forma adecuada de aplicación mediante casos prácticos, lo cual resulta mucho más didáctico que la difusión por los medios de comunicación que utiliza la organización. 
En función de los resultados anteriores, la corporación debe reorientar sus esfuerzos para disponer de un modelo de gestión abocado a la calidad total de sus operaciones, es decir trabajar por la consolidación de un sistema de gestión que asuma como principio la calidad plena de sus operaciones de servicios.

En este sentido, se precisan como elementos de lo que pudiera ser un modelo de gestión de la calidad para la corporación, elementos asociados con las responsabilidades de la dirección y la gestión que estos realizan de los recursos disponibles.

Al estudiar estos elementos en la corporación, se tiene para la Responsabilidad de la Dirección, que es obligación de la parte directiva de la Corporación Nacional de Telecomunicaciones CNT EP, la toma de decisiones eficientes, a fin de alcanzarlas políticas y lineamientos internos de la organización. Bajo su responsabilidad se encuentra, el velar por los intereses de la empresa, así como buscar en todo momento, que las actividades que se desarrollan para la prestación de servicios, cumplan los parámetros de calidad contemplados en el Manual de Calidad de la empresa.

Cabe además indicar que su gestión debe estar enfocada en el marco de la responsabilidad, no solo con la empresa sino también con sus grupos de interés como son clientes, proveedores, competencia, medio ambiente, entre otros, con dicha gestión se pretende satisfacer las necesidades y expectativas de estos actores. Deben además garantizar un clima laboral saludable, generando compromiso y empoderamiento con sus colaboradores, lo cual se refleja en el éxito de la organización.

Con respecto a la alta dirección de la corporación, esta juega un papel preponderante al revisar y asegurar la disponibilidad y asignación de recursos necesarios para el desarrollo de las actividades de la empresa, procurando alcanzar la mejora continua de sus procesos, enfocado en satisfacer las necesidades de los clientes mediante la prestación de servicios y productos con altos estándares de calidad. Se encargan de revisar resultados de la empresa para presentarlos al Directorio. Deben cerciorarse sobre el cumplimiento de la Política de la Calidad y sus objetivos a fin de obtener la satisfacción de los clientes.

El compromiso de los líderes estratégicos de esta compañía, debe orientarse al cliente, lo que asegura que tanto los productos como los servicios que brinda la corporación se incluyan en la guía comercial y que sus requisitos de contratación sean respetados y comprendidos por los demandantes del mercado. Debe prestar especial atención a los factores que disminuyen el nivel de satisfacción de los clientes, así como la calidad de los productos y servicios.

Si bien este enfoque de liderazgo contempla lo detallado en párrafos anteriores, se plantea que se descentralice en el sentido de que por su alta carga laboral los directivos no pueden estar completamente inmiscuidos en todos estos aspectos, se debe delegar responsabilidad 
a otros niveles jerárquicos como los jefes departamentales de cada provincia a fin de que sean ellos los encargados de monitorear el nivel de satisfacción de los usuarios de la CNT EP.

Con respecto a la Gestión de Recursos, su administración debe enfocarse en gestionar recursos que permitan cumplir la planeación estratégica de manera eficiente y además considerar que se debe gestionar recursos para solventar imprevistos que puedan presentarse por alguna situación. Al contar con los recursos, resulta mucho más fácil emprender actividades y planes de acción enfocados en los procesos de mejora continua que beneficien a la corporación y sus miembros. Al hablar de recursos, no solo se hace referencia a la parte económica, sino también se debe considerar la gestión del recurso humano a fin de que sea suficiente y se encuentre capacitado para el desarrollo de actividades que le han sido encomendadas.

Con respecto a la obtención del servicio, los recursos obtenidos por la Dirección de la empresa, deben estar encaminados a la obtención de servicios, es decir se pasa de planificar a hacer, para lo cual es necesario realizar la inversión de recursos en necesidades propias de la empresa, dando prioridad a las más relevantes. Los productos y servicios que son comercializados por la Corporación Nacional de Telecomunicaciones CNT EP, deben cumplir estándares de calidad a fin de satisfacer las necesidades de sus clientes y consumidores.

Lo atinente a la medición, análisis y mejora, todo proceso que se lleve a cabo en la organización, debe ser medible, cuantificable y proyectarse a la mejora. Es decir, los bienes y servicios que brinda la Corporación Nacional de Telecomunicaciones CNT EP a sus clientes y consumidores, deben estar sujetos a medición, se puede medir la calidad del producto, el nivel de satisfacción que brinda a quien lo utiliza, la rentabilidad que genera para la organización y la eficiencia del proceso con la que se lleva a cabo la actividad.

En caso de ser detectado cualquier inconveniente o insatisfacción, se propone realizar identificar la causa del problema, a fin de solventarlo en el menor tiempo posible, es decir sin que produzca mayor afectación ni a la empresa, sus colaboradores y sus clientes. Cuando se tenga identificado el punto débil que está generando el conflicto, se implementa la mejora en caso de tener autonomía o de lo contrario se comunica a la parte directiva que será la encargada de evaluar la propuesta de mejora para su implementación y posterior evaluación a fin de garantizar que se cumpla lo planteado.

Los resultados anteriores permiten direccionar la organización hacia la prestación de un servicio de calidad, en constante revisión y análisis, donde la mejora continua ser el fin último perseguido como parte del cambio gradual que se debe ser asumido por esta importante Corporación de la ciudad de Azogues, Ecuador. 
Este modelo de gestión y su implementación, pasa por considerar la calidad como lineamiento central que transverzalice todos los servicios prestados por la Corporación en bienestar tanto de los usuarios, clientes y la comunidad en general.

\section{Modelo de Gestión de la Calidad en la Corporación Nacional de Telecomunicaciones CNT EP: hacia la mejor continua.}

La propuesta de este trabajo de investigación es un Modelo de gestión de la calidad para la mejora Continua para la calidad del servicio de internet en la Unidad Comercial de la Corporación Nacional de Telecomunicaciones CNT EP en la Ciudad de Azogues. Es necesario conocer la organización, operación de procesos con sus riesgos y planes de contingencia, aspectos técnicos, leyes y reglamentos, requerimientos de clientes y de mercado; y, el direccionamiento estratégico; los cuales se encuentran en documentos de la CNT EP. En caso de requerir conocimientos adicionales se los impartirá mediante capacitaciones específicas.

Todos los colaboradores de la CNT EP a nivel nacional deben tener conciencia sobre la importancia de la Política de Gestión de la Calidad y sus objetivos mediante la difusión y sensibilización que implica no cumplir los requisitos del Sistema de Gestión de la Calidad. Se debe comprometer a los miembros de la organización a fin de garantizar su cumplimiento eficiente.

Se debe mantener completamente informado al personal sobre el Sistema de Gestión de la Calidad, cuya información se da a conocer a través del sistema de información documental MAI (manejo automático de la ISO) mediante el cual se sociabiliza sobre los procesos, funciones y responsabilidades.

El conocer la situación actual de la empresa, sirve de base para diseñar el modelo de mejora continua para la calidad del servicio de internet en la Unidad Comercial de la Corporación Nacional de Telecomunicaciones CNT EP en la Ciudad de Azogues, está enfocado en el servicio de internet fijo residencial por cobre que se brinda en la Parroquia Javier Loyola, se fundamenta en las debilidades encontradas en la evaluación de la satisfacción del cliente, necesidades internas del personal, entre otras y en el análisis previo realizado (Entrevistas, 2018); se plantea mejorar el servicio y satisfacer los requerimientos de los consumidores, para lo cual se deberá garantizar un servicio de calidad, que incluya excelente atención al cliente, servicio posventa y atención de reclamos por daños técnicos y de facturación acorde a las expectativas de los clientes.

A continuación, se representa gráficamente el modelo de mejora continua para la calidad del servicio de internet en la Unidad Comercial de la Corporación Nacional de Telecomunicaciones CNT EP en la Ciudad de Azogues. 
Figura 2. Propuesta de Modelo de Gestión de la Calidad para la Corporación Nacional de Telecomunicaciones CNT EP

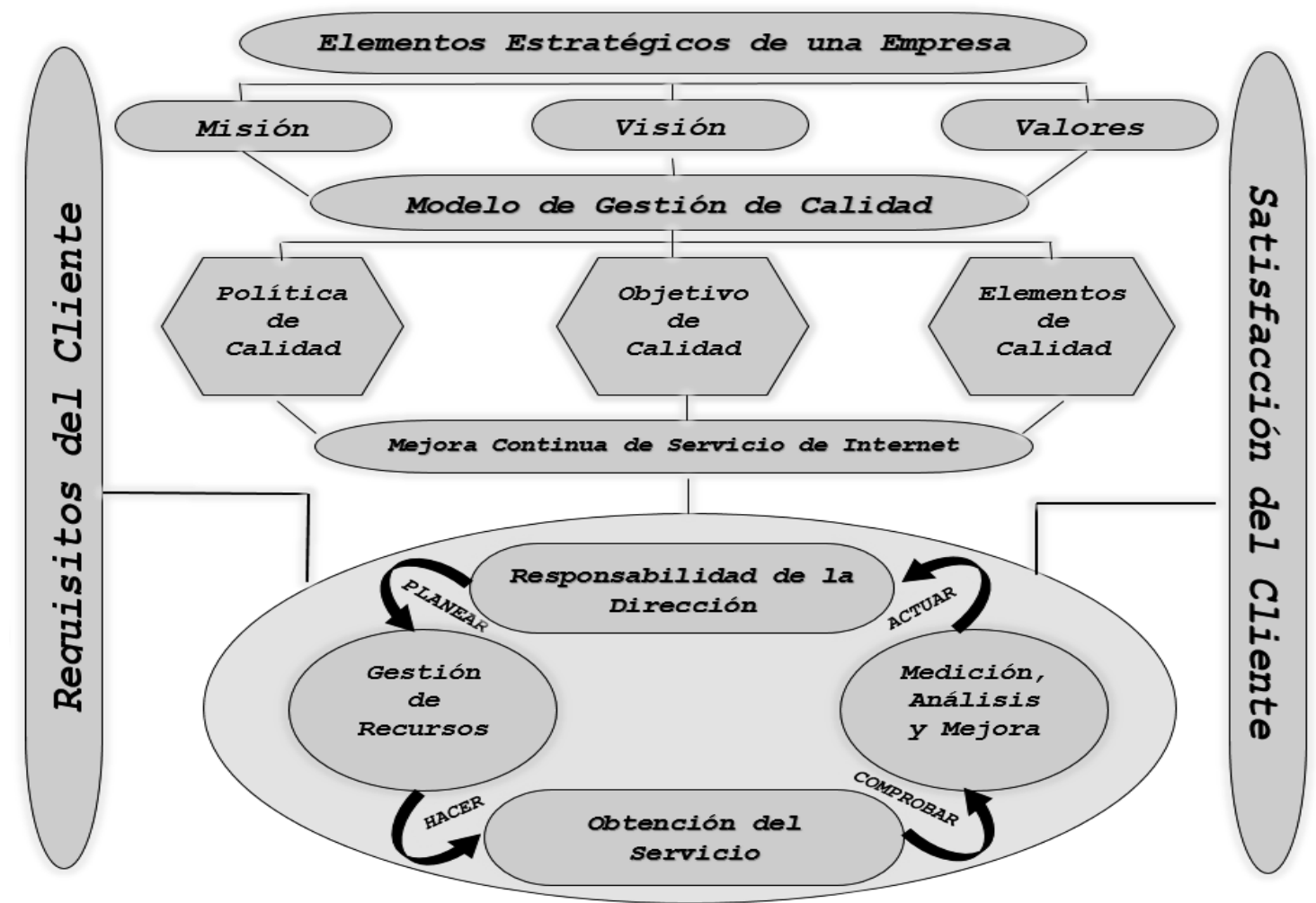

Fuente: Elaboración propia

El proceso de mejora continua en los sistemas de gestión de la calidad, se ha convertido en una muy valiosa herramienta aplicada por las organizaciones en busca de la eficiencia y efectividad en su gestión, lo cual conduce al éxito. Tanto la calidad como el servicio y la mejora continua se convierten en un factor de interés público, es decir capta el interés de las empresas y los clientes, donde a más de buscar rentabilidad, sea esta económica o social, se pretende satisfacer las necesidades y requerimientos de los usuarios y consumidores de bienes y servicios.

\section{Conclusiones.}

- Las responsabilidades de ejecución de planes y nuevas políticas de mejora a la calidad del portafolio de servicios que posee la Corporación Nacional de Telecomunicaciones CNT EP y de forma particular para el internet, estuvieron designados de acuerdo al Reglamento Orgánico Funcional a altos funcionarios que laboran en la ciudad de Quito, marginando la participación a los jefes departamentales de la CNT EP- Azogues en la ejecución, implementación, evaluación de los planes y, al monitoreo de los mismos a nivel de satisfacción de los usuarios. De la misma forma, la Política de la Calidad no fue cumplida por todos los colaboradores de la empresa. 
- La infraestructura del servicio de internet con el sistema por cobre que ofreció la Corporación Nacional de Telecomunicaciones CNT EP en la Parroquia Javier Loyola de la Ciudad de Azogues, presentó fallas por la obsolescencia y saturación de la red, ocasionando baja velocidad en el servicio y obstáculos para la empresa; incidentes que no fueron atendidos de forma apropiada y eficiente por Unidad Técnica, encargada de la atención de daños.

- La formulación y estructura del modelo de mejora continua para la calidad del servicio de internet, fue diseñado a partir de diagnóstico realizado en la corporación, elaborada para el propósito, el cual mantiene su alineamiento con el Modelo de Gestión de la Calidad ISO 9001-2015 y promueve resultados favorables para la empresa en corto, mediano y largo plazo.

- Con la propuesta del modelo de mejora continua para la calidad del servicio de internet en la Unidad Comercial de la Corporación Nacional de Telecomunicaciones CNT EP en la Ciudad de Azogues, el servicio de internet que brinda la corporación, puede orientarse a cumplir con las exigencias y necesidades de clientes y generar entre los colaboradores la cultura de servicio al cliente, quien debe ser atendido de manera eficiente y oportuna, todos los funcionarios deben aplicar la política de calidad e la empresa.

- Se debe gestionar ante los directivos de la organización, la contratación de personal técnico para que solvente daños en el servicio de internet, que no pueden ser cubiertos en su totalidad por el personal, este retraso en la atención causa malestar entre los clientes y usuarios del servicio, generando insatisfacción.

- Se considera oportuno que ante la saturación de la red de cobre en ciertas localidades y especialmente en la Parroquia Javier Loyola, se gestione el abastecimiento de infraestructura por fibra óptica.

- Se recomienda evaluar los resultados obtenidos con la implementación del modelo y seguir mejorando hasta lograr la mayor satisfacción de los clientes en el menor tiempo posible y cumplimiento del objetivo propuesto.

- Una vez implementada la propuesta, se deben revisar periódicamente no solo los procesos sino también las funciones de cada operador (directivo, funcionario, empleado), ya que si realizan funciones que no le competen y para lo cual no ha sido capacitado, reducirá la producción individual.

- Proporcionar y socializar el modelo a los Directivos de la Corporación Nacional de Telecomunicaciones CNT EP de Azogues, para de ser el caso, se aplique el mismo a partir del año 2019. 


\section{Referencias Bibliográficas.}

Aja, L. (2002). Gestión de información, gestión del conocimiento y gestión de la calidad en las organizaciones. Acimed, 10(5), 7-8. Recuperado de http://scielo.sld.cu/scielo.php?script=sci_arttext\&pid=S102494352002000500004

Bernal, I., Pedraza, N., y Sánchez, M. (2015). El clima organizacional y su relación con la calidad de los servicios públicos de salud: diseño de un modelo teórico. Estudios Gerenciales, $\quad 31(134), \quad 8-19 . \quad$ Recuperado de http://www.redalyc.org/articulo.oa?id=21233043002

Cortez, S. (2015). Estrategias de calidad como instrumento parael desarrollo de la competitividad de las micro y pequeñas empresas de la Ciudad de Trujillo (tesis doctoral). Universidad Nacional de Trujillo, Trujillo, Perú.

Cuesta, M., y Isaac, C. (2008) Metodología para la mejora de los procesos del sistema de gestión de la calidad de la gerencia de proyectos de Etecsa. Ingeniería Industrial, 29(3), 1-7. Recuperado de http://www.redalyc.org/articulo.oa?id=360433567008

Feigenbaum, A. (1994). Control total de la calidad. México; Compañía Editorial Continental.

García, M., Quispe, C., y Ráez, L. (2003). Mejora continua de la calidad en los procesos. Industrial Data,6(1), 89-94. Recuperado de http://www.redalyc.org/articulo.oa?id=81606112

González, A., y Fernández, E. (2000). Diseño de un modelo para desarrollar los proyectos de mejora continua de la calidad. Ensaios e Ciência: Ciências Biológicas, Agrárias e da Saúde, 4(3), 55-67. Recuperado de http://www.redalyc.org/articulo.oa?id=26040304

Gutierrez, A. (2004). La calidad en el servicio. Revista de Enfermería del Instituto Mexicano del Seguro Social, 12(1), 1-2. Recuperado de ww.medigraphic.com/pdfs/enfermeriaimss/eim-2004/eim041a.pdf

Huerta, J., y Gómez, R. (2013). Concentración y diversidad de los medios de comunicación y las telecomunicaciones en México. Comunicación y sociedad. (19), 113-152. Recuperado de http://www.scielo.org.mx/pdf/comso/n19/n19a6.pdf

Isaza, A. (2014). Control interno y sistema de gestión de calidad. Bogotá, Colombia: Ediciones de la U.

James, P. T. (1997). Gestión de la Calidad Total. Un texto Introductorio. España. 
Prentice Hall,

Jiménez, C., Argueta, G., y Espinoza, B. (2014). Revisión de literatura sobre mejora continua en Mipymes iberoamericanas. Nóesis. Revista de Ciencias Sociales y Humanidades, 23(46), 140-162. Recuperado de http://www.redalyc.org/articulo.oa?id=85930565006

León, C., Iturri, L., y León, C. (2007). Mejora continua para el sistema de Comunicaciones. Polibits, (36), 25-29. Recuperado de http://www.redalyc.org/articulo.oa?id=402640449005

Lizarzaburu, E. (2016). La gestión de la calidad en Perú: un estudio de la norma ISO 9001, sus beneficios y los principales cambios en la versión 2015. Universidad \& Empresa, 18(30), 33-54. Recuperado de https://revistas.urosario.edu.co/index.php/empresa/article/view/4604

López, P. (2004). Población muestra y muestreo. Punto Cero, 9(8), 69-74. Recuperado de http://www.scielo.org.bo/scielo.php?script=sci_arttext\&pid=S181502762004000100012

Marín, J., Bautista, Y., y García, J. (2014). Etapas en la evolución de la mejora continua: estudio multicaso. Intangible capital, 10(3), 584-618. Recuperado de http://www.redalyc.org/articulo.oa?id=54932488008

Membrado, J., (2002), Innovación y mejora continua según el Modelo EFQM de excelencia. https://books.google.com.ec/books?id=Y40klEWbNwEC\&pg=PA269\&dq=mem brado+2002\&hl=es\&sa=X\&ved=0ahUKEwiytO6DjrbfAhVE11kKHZARBR8Q $6 A E I J z A A \# v=$ onepage $\& q=$ membrado $\% 202002 \& \mathrm{f}=$ false

Parasuraman, A., Zeithalm, V., y Berry, L. (1985). A Conceptual Model of Service Quality and Its Implications for Future Research. Journal of Marketing, 49(4), 4150.

https://www.jstor.org/stable/1251430?seq=1\#page_scan_tab_contents

Peña, N. (1992). Gerencia del servicio. Innovar, 2(2), 65-66. Recuperado de https://revistas.unal.edu.co/index.php/innovar/article/view/21631/22627

Quecedo, R., y Castaño, C. (2002). Introducción a la metodología de investigación cualitativa. Revista de Psicodidáctica, (14), 5-39. Recuperado de http://www.redalyc.org/articulo.oa?id=17501402 
Romero, D. (2017). Gestión de calidad enfocado a procesos para mejorar el nivel de servicio de resolución de reclamos en el área de back office de la empresa bpo consulting S.A.C. Independencia 2016 (Tesis de pregrado). Univesidad César Vallejo, Lima, Perú.

Schneider, G. (2016). El servicio universal: la garantía de un suministro básico de servicios de telecomunicaciones.Revista Jurídica Universidad Autónoma de Madrid, (2), 215-236. Recuperado de https://revistas.uam.es/revistajuridica/article/view/6287/6759

Soto, A. (2013). Calidad en la atención al cliente en las pymes del Municipio de Arauca (Tesis de maestría). Universidad Nacional de Colombia, Arauca, Colombia.

Vargas, M., y Aldana, L. (2014). Calidad y servicio conceptos y herramientas. Bogotá, Colombia: Ecoe Ediciones. 
Para citar el artículo indexado.

Torres A, Luna K., Ormaza J. \& Cantos M. (2019). Gestión de la calidad en el sector de telecomunicaciones. Orientaciones hacia la mejora continua en la Corporación Nacional de Telecomunicaciones, Azogues - Ecuador. Revista electrónica Visionario Digital 3(2), 170-190. Recuperado desde:

http://www.cienciadigital.org/revistascienciadigital/index.php/VisionarioDigital/article/view/4 $\underline{07 / 925}$

\section{Ciencia \\ LDigital}

El artículo que se publica es de exclusiva responsabilidad de los autores y no necesariamente reflejan el pensamiento de la Revista Ciencia Digital.

El articulo queda en propiedad de la revista y, por tanto, su publicación parcial y/o total en otro medio tiene que ser autorizado por el director de la Revista Ciencia Digital.
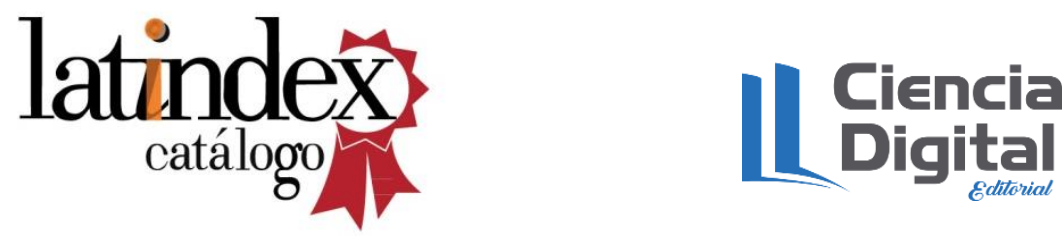